PROCEEDINGS OF THE

AMERICAN MATHEMATICAL SOCIETY

Volume 138, Number 3, March 2010, Pages 899-906

S 0002-9939(09)10200-9

Article electronically published on November 5, 2009

\title{
ON CONVEX TO PSEUDOCONVEX MAPPINGS
}

\author{
S. IVASHKOVICH
}

(Communicated by Franc Forstneric)

\begin{abstract}
In the works of Darboux and Walsh, it was remarked that a one-toone self-mapping of $\mathbb{R}^{3}$ which sends convex sets to convex ones is affine. It can be remarked also that a $\mathcal{C}^{2}$-diffeomorphism $F: U \rightarrow U^{\prime}$ between two domains in $\mathbb{C}^{n}, n \geq 2$, which sends pseudoconvex hypersurfaces to pseudoconvex ones is either holomorphic or antiholomorphic.

In this paper we are interested in the self-mappings of $\mathbb{C}^{n}$ which send convex hypersurfaces to pseudoconvex ones. Their characterization is the following: $A$ $\mathcal{C}^{2}$-diffeomorphism $F: U^{\prime} \rightarrow U$ (where $U^{\prime}, U \subset \mathbb{C}^{n}$ are domains) sends convex hypersurfaces to pseudoconvex ones if and only if the inverse map $\Phi:=F^{-1}$ is weakly pluriharmonic, i.e., if it satisfies some nice second order PDE very close to $\partial \bar{\partial} \Phi=0$. In fact all pluriharmonic $\Phi$ 's do satisfy this equation, but there are also other solutions.
\end{abstract}

\section{Formulation}

Let $U^{\prime}, U$ be domains in $\mathbb{C}^{n}, n \geq 2$ and let $F: U^{\prime} \rightarrow U$ be a $\mathcal{C}^{2}$-diffeomorphism. Coordinates in the source we denote by $z^{\prime}=x^{\prime}+i y^{\prime}$, in the target by $z=x+i y$. It will be convenient for us to suppose that $U^{\prime}$ is a convex neighborhood of zero and that $F\left(0^{\prime}\right)=0$. The somewhat unusual choice to put primes on the objects in the source (and not in the target) is explained by the fact that in the statements and in the proofs we shall work more with the inverse map $\Phi$ than with $F$.

Theorem. Let $F: U^{\prime} \rightarrow U$ be a $\mathcal{C}^{2}$-diffeomorphism. Then the following conditions are equivalent:

i) For every convex hypersurface $M^{\prime} \subset U^{\prime}$ the image $M=F\left(M^{\prime}\right)$ is a pseudoconvex hypersurface in $U$.

ii) The inverse map $\Phi:=F^{-1}: U \rightarrow U^{\prime}$ satisfies the following PDE:

$$
\partial \bar{\partial} \Phi=\left(d \Phi^{-1}(\Delta \Phi), d z\right) \wedge \partial \Phi+\left(d z, d \Phi^{-1}(\Delta \Phi)\right) \wedge \bar{\partial} \Phi .
$$

iii) For every $z \in U$ and every $\zeta \in T_{z} \mathbb{C}^{n}$ one has

$$
\partial \bar{\partial} \Phi_{z}(\zeta, \bar{\zeta}) \in \operatorname{span}\left\{d \Phi_{z}(\zeta), d \Phi_{z}(i \zeta)\right\} .
$$

Here we use the following notation: for a vector $v=\left(v^{1}, \ldots, v^{n}\right) \in \mathbb{C}^{n}$ and $d z=\left(d z_{1}, \ldots, d z_{n}\right)$ we set $(d z, v)=\bar{v}^{j} d z_{j}$ and $(v, d z)=v^{j} d \bar{z}_{j}$. Throughout this paper we shall use the Einstein summation convention.

Received by the editors March 10, 2009.

2010 Mathematics Subject Classification. Primary 32F10; Secondary 52A20, 32 U15.

Key words and phrases. Convex, pseudoconvex, pluriharmonic.

(C)2009 American Mathematical Society 
Remark. 1. Pluriharmonic $\Phi$ 's clearly satisfy (1.1) (or (1.2)); let us remark that this geometric characterization of pluriharmonic diffeomorphisms perfectly agrees with an analytic one: The class $\mathcal{P}$ of pluriharmonic diffeomorphisms $\mathbb{C}^{n} \rightarrow \mathbb{C}^{n}$ is stable under biholomorphic parametrization of the source and $\mathbb{R}$-linearization of the target. Really, these parametrizations preserve accordingly pseudoconvexity and convexity of hypersurfaces.

2. Item (i) of the theorem is clearly equivalent to the following: For every strictly convex quadric $M^{\prime} \cap U^{\prime} \neq \emptyset$, the image $M=F\left(M^{\prime} \cap U^{\prime}\right)$ is a pseudoconvex hypersurface in $U$; i.e, it is enough to check this condition only for quadrics.

3. The fact that (1.1) admits solutions other than just pluriharmonic mappings is very easy to see from the form of its linearization at the identity:

$$
\partial \bar{\partial} \Phi=(\Delta \Phi, d z) \wedge d z .
$$

Observe that any map of the form $\Phi(z)=\left(\phi_{1}\left(z_{1}\right), \ldots, \phi_{n}\left(z_{n}\right)\right)$ satisfies $(1.3)$ provided all $\phi_{j}$, except for some $j_{0}$, are harmonic. This $\phi_{j_{0}}$ can then be an arbitrary $\mathcal{C}^{2}$ function.

4. One can consider the relation (1.2) as one that gives a geometric meaning to equation (1.1).

\section{AN AUXILIARY COMPUTATION}

Denote by $\zeta=\xi+i \eta$ a tangent vector at point $z \in \mathbb{C}^{n}$. Recall that the real Hessian of a real-valued function $\rho$ in $\mathbb{C}^{n}=\mathbb{R}^{2 n}$ is

$$
H_{\rho(z)}^{\mathbb{R}}(\zeta, \zeta)=\frac{\partial^{2} \rho(z)}{\partial x_{i} \partial x_{j}} \xi_{i} \xi_{j}+\frac{\partial^{2} \rho(z)}{\partial y_{i} \partial y_{j}} \eta_{i} \eta_{j}+2 \frac{\partial^{2} \rho(z)}{\partial x_{i} \partial y_{j}} \xi_{i} \eta_{j} .
$$

A hypersurface $M=\{z \in U: \rho(z)=0\}$, with $\rho$ being $\mathcal{C}^{2}$-regular, $\rho(0)=0$ and $\left.\nabla \rho\right|_{M} \neq 0$, is strictly convex if the defining function $\rho$ can be chosen with positive definite Hessian, i.e., $H_{\rho(z)}^{\mathbb{R}}(\zeta, \zeta)>0$ for all $z \in M$ and all $\zeta \neq 0$. One readily checks the following expression of the real Hessian of $\rho$ in complex coordinates:

$$
H_{\rho(z)}^{\mathbb{R}}(\zeta, \zeta)=\frac{\partial^{2} \rho(z)}{\partial z_{i} \partial z_{j}} \zeta_{i} \zeta_{j}+\frac{\partial^{2} \rho(z)}{\partial \bar{z}_{i} \partial \bar{z}_{j}} \bar{\zeta}_{i} \bar{\zeta}_{j}+2 \frac{\partial^{2} \rho(z)}{\partial z_{i} \partial \bar{z}_{j}} \zeta_{i} \bar{\zeta}_{j}
$$

Recall that the Hermitian part $L_{\rho(z)}(\zeta, \bar{\zeta})=\frac{\partial^{2} \rho}{\partial z_{i} \partial \bar{z}_{j}} \zeta_{i} \bar{\zeta}_{j}$ of the Hessian is called the Levi form of $\rho$ (and of $M$ ). $M$ is strictly pseudoconvex if its Levi form is positive definite on the complex tangent space $T_{z}^{c} M=\left\{\zeta \in T_{z} \mathbb{C}^{n}:(\bar{\partial} \rho(z), \zeta)=0\right\}$ for every $z \in M$. Here $(\cdot, \cdot)$ stands for the standard Hermitian scalar product in $\mathbb{C}^{n}$.

Let $F: \mathbb{C}_{z^{\prime}}^{n} \supset U^{\prime} \rightarrow U \subset \mathbb{C}_{z}^{n}$ be a $\mathcal{C}^{2}$-diffeomorphism. Further, let $z^{\prime}=z^{\prime}(z)$ be the coordinate representation of the inverse mapping $z^{\prime}=\Phi(z):=F^{-1}(z)$ and let $M=F\left(M^{\prime}\right) \subset U$ be the image of a hypersurface $M^{\prime} \subset U^{\prime}$. Then $M=\{z: \rho(z)=$ $0\}$, where $\rho(z):=\rho^{\prime}\left(z^{\prime}(z)\right)$.

Lemma 2.1. The Levi form of $\rho$ at point $z$ decomposes as

$$
L_{\rho(z)}(\zeta, \bar{\zeta})=L_{\rho(z)}^{0}(\zeta, \bar{\zeta})+L_{\rho(z)}^{1}(\zeta, \bar{\zeta})
$$

where

$$
L_{\rho(z)}^{0}(\zeta, \bar{\zeta})=\frac{1}{4} H_{\rho^{\prime}\left(z^{\prime}\right)}^{\mathbb{R}}\left(d \Phi_{z}(\zeta), d \Phi_{z}(\zeta)\right)+\frac{1}{4} H_{\rho^{\prime}\left(z^{\prime}\right)}^{\mathbb{R}}\left(d \Phi_{z}(i \zeta), d \Phi_{z}(i \zeta)\right)
$$


and

$$
L_{\rho(z)}^{1}(\zeta, \bar{\zeta})=2\left\langle\nabla \rho^{\prime}\left(z^{\prime}\right), \partial \bar{\partial} \Phi_{z}(\zeta, \bar{\zeta})\right\rangle=2 \operatorname{Re}\left(\bar{\partial} \rho^{\prime}\left(z^{\prime}\right), \partial \bar{\partial} \Phi_{z}(\zeta, \bar{\zeta})\right)
$$

Proof. Here we denote by $d \Phi_{z}$ the differential of the inverse map $\Phi:=F^{-1}$ at the point $z, \nabla \rho^{\prime}\left(z^{\prime}\right)$ the real gradient of $\rho^{\prime}$ at $z^{\prime}$, and $\langle\cdot, \cdot\rangle=\operatorname{Re}(\cdot, \cdot)$ the standard Euclidean scalar product in $\mathbb{C}^{n}$.

Denote by $\nu$ the vector with components $\nu_{j}=\frac{\partial z_{j}^{\prime}}{\partial z_{\alpha}} \zeta_{\alpha}$ and by $\mu$ the vector with $\mu_{j}=\frac{\partial z_{j}^{\prime}}{\partial \bar{z}_{\alpha}} \bar{\zeta}_{\alpha}$, i.e.,

$$
\nu=\partial \Phi_{z}(\zeta) \text { and } \mu=\bar{\partial} \Phi_{z}(\zeta)
$$

Remark that

$$
\nu+\mu=d \Phi_{z}(\zeta) \text { and } i(\nu-\mu)=d \Phi_{z}(i \zeta)
$$

Write

$$
\begin{gathered}
L_{\rho(z)}(\zeta, \bar{\zeta})=\frac{\partial^{2} \rho}{\partial z_{\alpha} \partial \bar{z}_{\beta}} \zeta_{\alpha} \bar{\zeta}_{\beta}=\frac{\partial}{\partial z_{\alpha}}\left(\frac{\partial \rho^{\prime}}{\partial z_{i}^{\prime}} \frac{\partial z_{i}^{\prime}}{\partial \bar{z}_{\beta}}+\frac{\partial \rho^{\prime}}{\partial \bar{z}_{i}^{\prime}} \frac{\partial \bar{z}_{i}^{\prime}}{\partial \bar{z}_{\beta}}\right) \zeta_{\alpha} \bar{\zeta}_{\beta}=\frac{\partial^{2} \rho^{\prime}}{\partial z_{i}^{\prime} \partial z_{j}^{\prime}} \\
\cdot \frac{\partial z_{i}^{\prime}}{\partial \bar{z}_{\beta}} \frac{\partial z_{j}^{\prime}}{\partial z_{\alpha}} \zeta_{\alpha} \bar{\zeta}_{\beta}+\frac{\partial^{2} \rho^{\prime}}{\partial \bar{z}_{i}^{\prime} \partial \bar{z}_{j}^{\prime}} \frac{\partial \bar{z}_{i}^{\prime}}{\partial \bar{z}_{\beta}} \frac{\partial \bar{z}_{j}^{\prime}}{\partial z_{\alpha}} \zeta_{\alpha} \bar{\zeta}_{\beta}+\frac{\partial^{2} \rho^{\prime}}{\partial z_{i}^{\prime} \partial \bar{z}_{j}^{\prime}}\left(\frac{\partial z_{i}^{\prime}}{\partial \bar{z}_{\beta}} \frac{\partial \bar{z}_{j}^{\prime}}{\partial z_{\alpha}}+\frac{\partial z_{i}^{\prime}}{\partial z_{\alpha}} \frac{\partial \bar{z}_{j}^{\prime}}{\partial \bar{z}_{\beta}}\right) \zeta_{\alpha} \bar{\zeta}_{\beta} \\
+\left(\frac{\partial \rho^{\prime}}{\partial z_{i}^{\prime}} \frac{\partial^{2} z_{i}^{\prime}}{\partial z_{\alpha} \partial \bar{z}_{\beta}}+\frac{\partial \rho^{\prime}}{\partial \bar{z}_{i}^{\prime}} \frac{\partial^{2} \bar{z}_{i}^{\prime}}{\partial z_{\alpha} \partial \bar{z}_{\beta}}\right) \zeta_{\alpha} \bar{\zeta}_{\beta}=\frac{\partial^{2} \rho^{\prime}}{\partial z_{i}^{\prime} \partial z_{j}^{\prime}} \mu_{i} \nu_{j}+\frac{\partial^{2} \rho^{\prime}}{\partial \bar{z}_{i}^{\prime} \partial \bar{z}_{j}^{\prime}} \bar{\nu}_{i} \bar{\mu}_{j}+\frac{\partial^{2} \rho^{\prime}}{\partial z_{i}^{\prime} \partial \bar{z}_{j}^{\prime}} \\
\cdot\left[\mu_{i} \bar{\mu}_{j}+\nu_{i} \bar{\nu}_{j}\right]+\left(\frac{\partial \rho^{\prime}}{\partial z_{i}^{\prime}} \frac{\partial^{2} z_{i}^{\prime}}{\partial z_{\alpha} \partial \bar{z}_{\beta}}+\frac{\partial \rho^{\prime}}{\partial \bar{z}_{i}^{\prime}} \frac{\partial^{2} \bar{z}_{i}^{\prime}}{\partial z_{\alpha} \partial \bar{z}_{\beta}}\right) \zeta_{\alpha} \bar{\zeta}_{\beta}=L_{\rho(z)}^{0}(\nu, \mu)+L_{\rho(z)}^{1}(\zeta, \bar{\zeta})
\end{gathered}
$$

with

$$
L_{\rho(z)}^{0}(\nu, \mu)=\frac{\partial^{2} \rho^{\prime}}{\partial z_{i}^{\prime} \partial z_{j}^{\prime}} \nu_{i} \mu_{j}+\frac{\partial^{2} \rho^{\prime}}{\partial \bar{z}_{i}^{\prime} \partial \bar{z}_{j}^{\prime}} \bar{\nu}_{i} \bar{\mu}_{j}+\frac{\partial^{2} \rho^{\prime}}{\partial z_{i}^{\prime} \partial \bar{z}_{j}^{\prime}}\left[\mu_{i} \bar{\mu}_{j}+\nu_{i} \bar{\nu}_{j}\right]
$$

and

$$
L_{\rho(z)}^{1}(\zeta, \bar{\zeta})=\left(\frac{\partial \rho^{\prime}}{\partial z_{i}^{\prime}} \frac{\partial^{2} z_{i}^{\prime}}{\partial z_{\alpha} \partial \bar{z}_{\beta}}+\frac{\partial \rho^{\prime}}{\partial \bar{z}_{i}^{\prime}} \frac{\partial^{2} \bar{z}_{i}^{\prime}}{\partial z_{\alpha} \partial \bar{z}_{\beta}}\right) \zeta_{\alpha} \bar{\zeta}_{\beta}
$$

We need to get more information about the structure of both terms $L_{\rho}^{0}$ and $L_{\rho}^{1}$ of the Levi form. Let's prove that the following relation holds:

$$
L_{\rho(z)}^{0}(\nu, \mu)=\frac{1}{4} H_{\rho^{\prime}\left(z^{\prime}\right)}^{\mathbb{R}}(\nu+\mu, \nu+\mu)+\frac{1}{4} H_{\rho^{\prime}\left(z^{\prime}\right)}^{\mathbb{R}}(i(\nu-\mu), i(\nu-\mu)) \text {. }
$$

To see this we make the following change in (2.9):

$$
\mu_{j}=V_{j}+i W_{j}, \nu_{j}=V_{j}-i W_{j}
$$

or

$$
V=\frac{1}{2}(\nu+\mu)=\frac{1}{2} d \Phi_{z}(\zeta), W=\frac{i}{2}(\nu-\mu)=\frac{1}{2} d \Phi_{z}(i \zeta) .
$$


Then

$$
\begin{gathered}
L_{\rho(z)}^{0}(\nu, \mu)=\frac{\partial^{2} \rho^{\prime}}{\partial z_{i}^{\prime} \partial z_{j}^{\prime}}\left(V_{i}-i W_{i}\right)\left(V_{j}+i W_{j}\right)+\frac{\partial^{2} \rho^{\prime}}{\partial \bar{z}_{i}^{\prime} \partial \bar{z}_{j}^{\prime}}\left(\overline{V_{i}}+i \overline{W_{i}}\right)\left(\overline{V_{j}}-i \overline{W_{j}}\right) \\
+\frac{\partial^{2} \rho^{\prime}}{\partial z_{i}^{\prime} \partial \bar{z}_{j}^{\prime}}\left[\left(V_{i}+i W_{i}\right)\left(\overline{V_{j}}-i \overline{W_{j}}\right)+\left(V_{i}-i W_{i}\right)\left(\overline{V_{j}}+i \overline{W_{j}}\right)\right] \\
=\frac{\partial^{2} \rho^{\prime}}{\partial z_{i}^{\prime} \partial z_{j}^{\prime}}\left(V_{i} V_{j}+W_{i} W_{j}\right)+i \frac{\partial^{2} \rho^{\prime}}{\partial z_{i}^{\prime} \partial z_{j}^{\prime}}\left(V_{i} W_{j}-W_{i} V_{j}\right)+\frac{\partial^{2} \rho^{\prime}}{\partial \bar{z}_{i}^{\prime} \partial \bar{z}_{j}^{\prime}}\left(\overline{V_{i} V_{j}}+\overline{W_{i} W_{j}}\right) \\
+i \frac{\partial^{2} \rho^{\prime}}{\partial \bar{z}_{i}^{\prime} \partial \bar{z}_{j}^{\prime}}\left(\overline{W_{i} V_{j}}-\overline{V_{i} W_{j}}\right)+2 \frac{\partial^{2} \rho^{\prime}}{\partial z_{i}^{\prime} \partial \bar{z}_{j}^{\prime}}\left(V_{i} \overline{V_{j}}+W_{i} \overline{W_{j}}\right) \\
=H_{\rho^{\prime}\left(z^{\prime}\right)}^{\mathbb{R}}(V, V)+H_{\rho^{\prime}\left(z^{\prime}\right)}^{\mathbb{R}}(W, W) .
\end{gathered}
$$

We used the obvious relations $\frac{\partial^{2} \rho^{\prime}}{\partial z_{i}^{\prime} \partial z_{j}^{\prime}}\left(V_{i} W_{j}-W_{i} V_{j}\right)=0=\frac{\partial^{2} \rho^{\prime}}{\partial \bar{z}_{i}^{\prime} \partial \bar{z}_{j}^{\prime}}\left(\overline{W_{i} V_{j}}-\overline{V_{i} W_{j}}\right)$ and the complex expression of the real Hessian (2.2). Therefore

$$
L_{\rho(z)}^{0}(\nu, \mu)=H_{\rho^{\prime}\left(z^{\prime}\right)}^{\mathbb{R}}(V, V)+H_{\rho^{\prime}\left(z^{\prime}\right)}^{\mathbb{R}}(W, W) .
$$

From (2.10) and (2.11) we get the formula (2.4) of the lemma.

Remark. If the real Hessian of $\rho^{\prime}$ at $z^{\prime}$ is positive (resp. non-negative) definite, then the component $L_{\rho(z)}^{0}(\nu, \mu)$ of the Levi form of $\rho$ at $z=F\left(z^{\prime}\right)$ is also positive (resp. non-negative) definite for any $\mathcal{C}^{2}$-germ of a diffeomorphism $F$.

Now we turn to $L_{\rho}^{1}$. Note that in complex notation $\nabla \rho=\bar{\partial} \rho$, and the standard Euclidean scalar product $\langle\cdot, \cdot\rangle$ in $\mathbb{C}^{n}$ is equal to the real part of the Hermitian product $(\cdot, \cdot)$. Therefore from $(2.8)$ we get

$$
\begin{gathered}
L_{\rho(z)}^{1}(\zeta, \bar{\zeta})=\left(\frac{\partial \rho^{\prime}}{\partial z_{i}^{\prime}} \frac{\partial^{2} z_{i}^{\prime}}{\partial z_{\alpha} \partial \bar{z}_{\beta}}+\frac{\partial \rho^{\prime}}{\partial \bar{z}_{i}^{\prime}} \frac{\partial^{2} \bar{z}_{i}^{\prime}}{\partial z_{\alpha} \partial \bar{z}_{\beta}}\right) \zeta_{\alpha} \bar{\zeta}_{\beta} \\
=\overline{\left(\bar{\partial} \rho^{\prime}, \frac{\partial^{2} z^{\prime}}{\partial \bar{z}_{\alpha} \partial z_{\beta}} \bar{\zeta}_{\alpha} \zeta_{\beta}\right)}+\left(\bar{\partial} \rho^{\prime}, \frac{\partial^{2} z^{\prime}}{\partial \bar{z}_{\alpha} \partial z_{\beta}} \bar{\zeta}_{\alpha} \zeta_{\beta}\right)=\overline{\left(\bar{\partial} \rho^{\prime}, \partial \bar{\partial} \Phi_{z}(\zeta, \bar{\zeta})\right)} \\
+\left(\bar{\partial} \rho^{\prime}, \partial \partial \bar{\partial} \Phi_{z}(\zeta, \bar{\zeta})\right)=2 \operatorname{Re}\left(\bar{\partial} \rho^{\prime}, \partial \bar{\partial} \Phi_{z}(\zeta, \bar{\zeta})\right)=2\left\langle\nabla \rho^{\prime}, \partial \bar{\partial} \Phi_{z}(\zeta, \bar{\zeta})\right\rangle,
\end{gathered}
$$

which proves (2.5).

\section{Proof of THE THEOREM}

(i) $\Longleftrightarrow$ (iii).

We start with the proof of the geometric characterization of convex to pseudoconvex mappings given in (iii) of the theorem. By a complex (real) line in $\mathbb{C}^{n}$ we mean a 1-dimensional complex (real) subspace of $\mathbb{C}^{n}$ and likewise for the complex (real) plane. Take a complex line $l=\operatorname{span}\{\zeta\}$ in $T_{z} \mathbb{C}^{n}$ and let $\Pi^{\prime} \subset T_{z^{\prime}} \mathbb{C}^{n}$ be the real plane, the image of $l$ under $d \Phi_{z}$, i.e., $\Pi^{\prime}=\operatorname{span}\left\{d \Phi_{z}(\zeta), d \Phi_{z}(i \zeta)\right\}$. Let $l^{\prime}:=\partial \bar{\partial} \Phi_{z}(l)$ denote the real (!) line, i.e., the image of $l$ under the mapping

$$
\partial \bar{\partial} \Phi_{z}: \mathbb{C}_{z}^{n} \rightarrow \mathbb{C}_{z^{\prime}}^{n},
$$

defined as

$$
\zeta \mapsto \partial \bar{\partial} \Phi(\zeta, \bar{\zeta}):=\frac{\partial^{2} \Phi(z)}{\partial z_{\alpha} \bar{\partial} z_{\beta}} \zeta_{\alpha} \bar{\zeta}_{\beta}
$$

We consider $l^{\prime}$ as a real line in $T_{z^{\prime}} \mathbb{C}^{n}$. 
Lemma 3.1. Suppose we are given a diffeomorphism $F: U^{\prime} \rightarrow U$. Then $F$ sends convex quadrics to pseudoconvex hypersurfaces if and only if for every $z \in U$ and for all $l^{\prime}:=\partial \bar{\partial} \Phi_{z}(l)$ and $\Pi^{\prime}=d \Phi_{z}(l)$ as above one has $l^{\prime} \subset \Pi^{\prime}$.

Proof. Let us prove the "only if" assertion first. We may suppose that $z^{\prime}=0^{\prime}$. Take any strictly convex $M^{\prime}=\left\{\rho^{\prime}\left(z^{\prime}\right)=0\right\}$ defined by a $\mathcal{C}^{2}$-function $\rho^{\prime}$ with positive defined Hessian such that $T_{0^{\prime}} M^{\prime} \supset \Pi^{\prime}$. By $M$ denote the image $F\left(M^{\prime}\right)$.

Consider the following family of hypersurfaces in $U^{\prime}: M_{t}^{\prime}=\left\{z: \rho_{t}^{\prime}\left(z^{\prime}\right):=\rho^{\prime}\left(z^{\prime}\right)+\right.$ $\left.t\left\langle\nabla \rho^{\prime}\left(0^{\prime}\right), z^{\prime}\right\rangle=0\right\}, t \in \mathbb{R}, t \neq-1$. All $M_{t}^{\prime}$ are strictly convex (they have the same quadratic part as $M^{\prime}$ ), all pass through zero and $M_{0}^{\prime}=M^{\prime}$. In addition all $M_{t}^{\prime}$ are smooth at zero with $T_{0} M_{t}^{\prime}=T_{0^{\prime}} M^{\prime}$ for all $t \neq-1$, because $\nabla \rho_{t}^{\prime}\left(0^{\prime}\right)=(1+t) \nabla \rho^{\prime}\left(0^{\prime}\right)$. Moreover, if we take some $\zeta \in T_{0}^{c} M$, then $\zeta$ will remain complex tangent to all $M_{t}:=F\left(M_{t}^{\prime}\right)$ at zero because $T_{0} M_{t}=d F_{0^{\prime}}\left(T_{0^{\prime}} M_{t}^{\prime}\right)$ is the same for all $t$. From Lemma 2.1 we see that

$$
L_{\rho_{t}(0)}(\zeta, \bar{\zeta})=L_{\rho(0)}^{0}(\zeta, \bar{\zeta})+2(1+t)\left\langle\nabla \rho^{\prime}\left(0^{\prime}\right), \partial \bar{\partial} \Phi(0)(\zeta, \bar{\zeta})\right\rangle,
$$

because $L_{\rho_{t}}^{0}(0)(\zeta, \bar{\zeta})=L_{\rho}^{0}(0)(\zeta, \bar{\zeta})$ for all $t$ due to the fact that the coefficients of $L_{\rho}^{0}$ depend only on the second derivatives of $\rho^{\prime}$ at $0^{\prime}$ and on $d \Phi_{0}$.

Suppose $\left\langle\nabla \rho^{\prime}\left(0^{\prime}\right), \partial \bar{\partial} \Phi(0)(\zeta, \bar{\zeta})\right\rangle \neq 0$. Then taking an appropriate $t_{0}$ we can make $L_{\rho_{t}(0)}(\zeta, \bar{\zeta})=0$ because $L_{\rho(0)}^{0}(\zeta, \bar{\zeta})$ do not depend on $t$. Remark that $t_{0} \neq-1$ because $L_{\rho(0)}^{0}(\zeta, \bar{\zeta})>0$. Now we can deform $M_{t}^{\prime}$ letting $t$ run over a neighborhood of $t_{0} . M_{t}^{\prime}$ stays strictly convex while the Levi form of $M_{t}$ changes its sign on the vector $\zeta$, a contradiction with the assumed property of $F$. Therefore

$$
\left\langle\nabla \rho^{\prime}\left(0^{\prime}\right), \partial \bar{\partial} \Phi(0)(\zeta, \bar{\zeta})\right\rangle=0
$$

for every strictly convex $M^{\prime}=\left\{z^{\prime}: \rho^{\prime}\left(z^{\prime}\right)=0\right\}$ such that $T_{0^{\prime}} M^{\prime} \supset \Pi^{\prime}$. For any vector $v \in T_{0^{\prime}} \mathbb{C}^{n}$ orthogonal to $\Pi^{\prime}$ we can take a strictly convex hypersurface $M^{\prime}=\left\{z^{\prime}: \rho^{\prime}\left(z^{\prime}\right)=0\right\}$ such that $\nabla \rho^{\prime}\left(0^{\prime}\right)=v$. Therefore $\partial \bar{\partial} \Phi(0)(\zeta, \bar{\zeta})$ is orthogonal to every such $v$. So $l^{\prime}=\partial \bar{\partial} \Phi(0)(l) \subset \Pi^{\prime}$ and the "only if " assertion of the lemma is proved.

To prove the opposite direction take a convex quadric $M^{\prime}=\left\{z^{\prime}: \rho^{\prime}\left(z^{\prime}\right)=0\right\}$ and set $M=F\left(M^{\prime}\right)$. Let $\zeta \in T_{z}^{c} M$. Use again Lemma 2.1. The term $L_{\rho(z)}^{0}(\zeta, \bar{\zeta})$ is clearly positive. The term $L_{\rho(z)}^{1}(\zeta, \bar{\zeta})$ is zero because $\partial \bar{\partial} \Phi_{z}(\zeta, \bar{\zeta}) \in d \Phi_{z}(\langle\zeta\rangle) \subset$ $T_{z^{\prime}} M^{\prime}$.

Let us reformulate the result obtained as follows (and remark that the equivalence of (i) and (iii) in the theorem is proved):

Corollary 3.1. F sends convex quadrics to pseudoconvex hypersurfaces if and only if for every $z \in U$ and every vector $\zeta \in T_{z} \mathbb{C}^{n}$ the following holds:

$$
\partial \bar{\partial} \Phi_{z}(\zeta, \bar{\zeta}) \in \operatorname{span}\left\{d \Phi_{z}(\zeta), d \Phi_{z}(i \zeta)\right\} .
$$

For the convenience of future references let us formulate the above-mentioned statement about holomorphic mappings:

Corollary 3.2. $A \mathcal{C}^{2}$-diffeomorphism $F: U^{\prime} \rightarrow U$ sends pseudoconvex quadrics to pseudoconvex hypersurfaces if and only if $F$ is either holomorphic or antiholomorphic. 
Proof. This is well known but let us still give a proof. Suppose, for example, that $\Phi$ is antiholomorphic; then $\nu=0$ as defined in (2.6). Therefore (2.5) tells us that $L_{\rho(z)}^{1}\left(\zeta^{\prime}, \bar{\zeta}^{\prime}\right) \equiv 0$ in the representation (2.3). Now (2.7) shows that

$$
L_{\rho(z)}(\zeta, \bar{\zeta})=L_{\rho^{\prime}\left(z^{\prime}\right)}\left(\bar{\partial} \Phi_{z}(\zeta), \bar{\partial} \Phi_{z}(\zeta)\right)
$$

for every complex tangent $\zeta$. The conclusion follows.

Suppose that, vice versa, $F$ sends pseudoconvex quadrics to pseudoconvex hypersurfaces. (3.3) shows that $\partial \bar{\partial} \Phi_{z}(\zeta, \bar{\zeta})$ belongs to the plane span $\left\{d \Phi_{z}(\zeta), d \Phi_{z}(i \zeta)\right\}$ for all $\zeta \in \mathbb{C}_{z}^{n}$. Therefore for every $\zeta$ complex tangent to $M=\{\rho(z)=0\}$ the vector $\partial \bar{\partial} \Phi_{z}(\zeta, \bar{\zeta})$ is tangent to $M^{\prime}=\left\{\rho^{\prime}\left(z^{\prime}\right)=0\right\}$. Consequently $L_{\rho(z)}^{1}(\zeta, \bar{\zeta}) \equiv 0$ for any $\rho$. Apply (2.9) to the quadric

$$
\rho^{\prime}\left(z^{\prime}\right)=\sum_{j=1}^{n}\left(z_{j}^{2}+\bar{z}_{j}^{2}+\varepsilon\left|z_{j}\right|^{2}\right)+L(z)+\overline{L(z)}
$$

(where $\varepsilon>0$ and $L$ is a $\mathbb{C}$-linear form) and get

$L_{\rho(z)}^{0}(\zeta, \bar{\zeta})=\sum_{j=1}^{n}\left(\nu_{j} \mu_{j}+\bar{\nu}_{j} \bar{\mu}_{j}+\varepsilon\left|\nu_{j}\right|^{2}+\varepsilon\left|\mu_{j}\right|^{2}\right)=2 \operatorname{Re}\left(\sum_{j=1}^{n} \nu_{j} \mu_{j}\right)+\varepsilon\left(\|\nu\|^{2}+\|\mu\|^{2}\right)$.

Taking different linear forms $L$ in (3.4) we can deploy any $\zeta \in \mathbb{C}^{n}$ as a complex tangent; and therefore, if $\Phi$ is neither holomorphic nor antiholomorphic, we see from (2.6) that $\nu$ and $\mu$ can be taken to be arbitrary. But for arbitrarily taken $\nu$ and $\mu,(3.5)$ cannot be positive, a contradiction.

(iii) $\Longleftrightarrow$ (ii).

We shall need the following linear algebra lemma. Let $V$ and $W$ be $\mathbb{C}$-linear spaces. We suppose that on $V$ some Hermitian scalar product $(\cdot, \cdot)$ is fixed. Let $B(\zeta, \bar{\eta}): V \times V \rightarrow W$ be a sesquilinear map. Its trace is defined as $\operatorname{Tr} B=$ $\sum_{\alpha} B\left(e_{\alpha}, \bar{e}_{\alpha}\right)$ for an orthonormal frame in $(V,(\cdot, \cdot))$. Furthermore, let $C: V \rightarrow W$ be an $\mathbb{R}$-linear isomorphism. Denote by $C^{1,0}$ (resp. $C^{0,1}$ ) the complex linear (resp. antilinear) part of $C$.

Lemma 3.2. The following properties of the pair $(B, C)$ are equivalent:

$$
B(\zeta, \bar{\zeta}) \in \operatorname{span}\{C(\zeta), C(i \zeta)\} \text { for all } \zeta \in V,
$$

$$
B(\zeta, \bar{\eta})=\left(C^{-1}(\operatorname{Tr} B), \eta\right) C^{1,0}(\zeta)+\left(\zeta, C^{-1}(\operatorname{Tr} B)\right) C^{0,1}(\eta) \text { for all } \zeta, \eta \in V .
$$

Proof. Define the induced quadratic map $A: V \rightarrow V$ as $A(\zeta, \bar{\zeta})=C^{-1} \circ B(\zeta, \bar{\zeta})$. Note that $A$ is not sesquilinear in general. Note that the image of every complex line in $V$ under a quadratic map is a real line.

Write (3.6) in the form $A(\zeta, \bar{\zeta})=k(\zeta) \cdot \zeta$, where $k$ is a complex-valued function. One readily sees that $k(\lambda \zeta)=\bar{\lambda} k(\zeta)$. The polarization equality for $A$,

$$
A(\zeta+\eta, \bar{\zeta}+\bar{\eta})+A(\zeta-\eta, \bar{\zeta}-\bar{\eta})=2 A(\zeta, \bar{\zeta})+2 A(\eta, \bar{\eta}),
$$

gives

$$
k(\zeta+\eta)(\zeta+\eta)+k(\zeta-\eta)(\zeta-\eta)=2 k(\zeta) \zeta+2 k(\eta) \eta
$$


or, for complex independent vectors,

$$
k(\zeta+\eta)+k(\zeta-\eta)=2 k(\zeta) \text { and } k(\zeta+\eta)-k(\zeta-\eta)=2 k(\eta),
$$

which implies additivity of $k: k(\zeta+\eta)=k(\zeta)+k(\eta)$ for complex independent $\zeta, \eta$ and, therefore, for all. So $k$ is an antilinear form on $V$ and by the Riesz representation we obtain a vector $v$ such that $k(\zeta)=(v, \zeta)$ for all $\zeta \in V$ and therefore $A(\zeta, \bar{\zeta})=(v, \zeta) \zeta$ and consequently

$$
B(\zeta, \bar{\zeta})=C((v, \zeta) \zeta) \text { for all } \zeta \in V .
$$

Furthermore,

$$
\begin{gathered}
B(\zeta, \bar{\eta})+B(\eta, \bar{\zeta})=B(\zeta+\eta, \bar{\zeta}+\bar{\eta})-B(\zeta, \bar{\zeta})-B(\eta, \bar{\eta})=C((v, \zeta+\eta)(\zeta+\eta)) \\
-C((v, \zeta) \zeta)-C((v, \eta) \eta)=C((v, \eta) \zeta)+C((v, \zeta) \eta)
\end{gathered}
$$

and

$$
\begin{gathered}
-i B(\zeta, \bar{\eta})+i B(\eta, \bar{\zeta})=B(\zeta+i \eta, \bar{\zeta}-i \bar{\eta})-B(\zeta, \bar{\zeta})-B(\eta, \bar{\eta})=C((v, \zeta+i \eta)(\zeta+i \eta)) \\
-C((v, \zeta) \zeta)-C((v, \eta) \eta)=C(-i(v, \eta) \zeta)+C(i(v, \zeta) \eta) .
\end{gathered}
$$

Therefore

$$
2 B(\zeta, \bar{\eta})=C((v, \eta) \zeta)-i C(v, \eta) \zeta)+C((v, \zeta) \eta)+i C(i((v, \zeta) \eta) .
$$

So we obtain

$$
B(\zeta, \bar{\eta})=(v, \eta) C^{1,0}(\zeta)+(\zeta, v) C^{0,1}(\eta)
$$

In (3.9) set $\zeta=\eta=e_{\alpha}$. Then

$$
\operatorname{Tr} B=\sum_{\alpha} B\left(e_{\alpha}, \bar{e}_{\alpha}\right)=C^{1,0}\left(\sum_{\alpha}\left(v, e_{\alpha}\right)\right)+C^{0,1}\left(\sum_{\alpha}\left(v, e_{\alpha}\right)\right)=C(v) .
$$

Therefore $v=C^{-1}(\operatorname{Tr} B)$ and (3.7) is established.

The opposite implication is easy, because (3.7) says that if $\eta$ is taken to be equal to $\zeta$, then

$$
\begin{gathered}
B(\zeta, \bar{\zeta})=a C^{1,0}(\zeta)+\bar{a} C^{0,1}(\zeta)=a \frac{1}{2}(C(\zeta)-i C(i \zeta))+\bar{a} \frac{1}{2}(C(\zeta)+i C(i \zeta)) \\
=\operatorname{Re} a \cdot C(\zeta)+\operatorname{Im} a \cdot C(i \zeta) \in \operatorname{span}\{C(\zeta), C(i \zeta)\}
\end{gathered}
$$

We apply this lemma for $B=\partial \bar{\partial} \Phi_{z}: T_{z} \mathbb{C}^{n} \rightarrow T_{z^{\prime}} \mathbb{C}^{n}, C=d F_{z^{\prime}}^{-1}: T_{z^{\prime}} \mathbb{C}^{n} \rightarrow T_{z} \mathbb{C}^{n}$ and, as a result, $A=d F_{z^{\prime}} \circ \partial \bar{\partial} \Phi_{z}: T_{z} \mathbb{C}^{n} \rightarrow T_{z} \mathbb{C}^{n}$ for every $z=F\left(z^{\prime}\right)$ and we get

Corollary 3.3. A $\mathcal{C}^{2}$-diffeomorphism $F$ sends convex quadrics to pseudoconvex hypersurfaces if and only if

$$
\partial \bar{\partial} \Phi_{z}(\zeta, \bar{\eta})=\left(d F_{z^{\prime}}\left(\operatorname{Tr} \partial \bar{\partial} \Phi_{z}\right), \eta\right) \partial \Phi(\zeta)+\left(\zeta, d F_{z^{\prime}}\left(\operatorname{Tr} \partial \bar{\partial} \Phi_{z}\right)\right) \bar{\partial} \Phi(\eta)
$$

for all $z=F\left(z^{\prime}\right)$ and all $\zeta, \eta \in T_{z} \mathbb{C}^{n}$.

This is equivalent to (1.1). The theorem is proved. 


\section{REFERENCES}

[1] M. Darboux, Sur un théorème fondamental de la géométrie projective (Extrait d'une lettre à M. Klein), Math. Ann. 17, no. 1 (1880), 55-61. MR1510050

[2] P. Schöpf, Konvexitätstreue und Linearität von Abbildungen, Math. Z. 177, no. 4 (1981), 533-540.

[3] J.L. Walsh, On the transformation of convex point sets, Ann. of Math. (2) 22, no. 4 (1921), 262-266. MR:1502587

UfR de Mathématiques, Université de Lille-1, 59655 Villeneuve D'AscQ, France and - Institute of Applied Problems of Mechanics and Mathematics, National Academy of Sciences of Ukraine, Lviv, Naukova 3B, 79601 Ukraine

E-mail address: ivachkov@math.univ-lille1.fr 